\title{
Analyzing Emotion Expression in Singing via Flow Glottograms, Long-Term-Average Spectra, and Expert Listener Evaluation
}

\author{
*`Johan Sundberg, ¥Gláucia Laís Salomão, and §’||Klaus R. Scherer, *††Stockholm, Sweden, §Geneva, Switzerland, and \\ || Munich, Germany
}

\begin{abstract}
Background. Acoustic aspects of emotional expressivity in speech have been analyzed extensively during recent decades. Emotional coloring is an important if not the most important property of sung performance, and therefore strictly controlled. Hence, emotional expressivity in singing may promote a deeper insight into vocal signaling of emotions. Furthermore, physiological voice source parameters can be assumed to facilitate the understanding of acoustical characteristics.

Method. Three highly experienced professional male singers sang scales on the vowel /ae/ or /a/ in 10 emotional colors (Neutral, Sadness, Tender, Calm, Joy, Contempt, Fear, Pride, Love, Arousal, and Anger). Sixteen voice experts classified the scales in a forced-choice listening test, and the result was compared with long-term-average spectrum (LTAS) parameters and with voice source parameters, derived from flow glottograms (FLOGG) that were obtained from inverse filtering the audio signal.

Results. On the basis of component analysis, the emotions could be grouped into four "families", AngerContempt, Joy-Love-Pride, Calm-Tender-Neutral and Sad-Fear. Recognition of the intended emotion families by listeners reached accuracy levels far beyond chance level. For the LTAS and FLOGG parameters, vocal loudness had a paramount influence on all. Also after partialing out this factor, some significant correlations were found between FLOGG and LTAS parameters. These parameters could be sorted into groups that were associated with the emotion families.

Conclusions. (i) Both LTAS and FLOGG parameters varied significantly with the enactment intentions of the singers. (ii) Some aspects of the voice source are reflected in LTAS parameters. (iii) LTAS parameters affect listener judgment of the enacted emotions and the accuracy of the intended emotional coloring.

Key Words: Enacting-Loudness-Emotion families-Parameter groups-Classical tradition.
\end{abstract}

\begin{abstract}
INTRODUCTION
Compared with the relatively important number of studies on the nature of vocal expression of emotion in speech that generally use acoustic analyses of emotion portrayals by lay or professional actors (see, for reviews, ${ }^{1-5}$ ), few published studies can be found for emotional expression in singing $\left(\right.$ see $\left.^{6}\right)$. Scherer, Sundberg, Fantini, Trznadel, and Eyben $^{7}$ have reviewed the scarce literature in this domain and reported a first major study on how singers' emotional interpretation of a musical piece affects acoustic parameters in the sung vocalizations. The results showed robust vocal signatures for the emotions studied, consisting of a major contrast between sadness and tenderness on the one hand, and anger, joy, and pride on the other. The acoustic patterns differentiating these emotions are low vs high
\end{abstract}

\footnotetext{
Accepted for publication August 8, 2019

This research was supported in part by funds granted to K. R. Scherer by the European Research Council (ERC) under Advanced Grant PROPEREMO 230331.

From the *Department of Speech Music Hearing, School of Electrical Engineering and Computer Science, KTH, Stockholm, Sweden; †University College of Music Education Stockholm, Stockholm, Sweden; tDepartment of Linguistics, Stockholm University, Stockholm, Sweden; $\S$ Department of Psychology, University of Geneva, Geneva, Switzerland; and the $\|$ Department of Psychology, University of Munich, Munich, Germany.

Address correspondence and reprint requests to Johan Sundberg, Department of Speech Music Hearing, School of Electrical Engineering and Computer Science, KTH, 10044, Stockholm, Sweden. E-mail: jsu@kth.se

Journal of Voice, Vol. $\square \square$, No. $\mathbf{\square}$, pp. $\mathbf{\square}-\mathbf{\square}$

0892-1997

(C) 2019 The Voice Foundation. Published by Elsevier Inc. All rights reserved.

https://doi.org/10.1016/j.jvoice.2019.08.007
}

levels on the components of loudness, vocal dynamics, high perturbation variation, and a tendency for high lowfrequency energy. The authors explain this pattern by the high vs low power and arousal characteristics of the respective emotions represented by these acoustic components.

While there is accumulating evidence for the acoustic parameters of emotional singing, documented in terms of long-term-average spectra, so far, there has been little effort to study the underlying mechanisms in voice production. In particular, given the technical difficulties, there has been little work on the physiological contributions as reflected in the pulsating glottal airflow as represented in terms of a flow glottogram (FLOGG). This airflow can be derived by inverse filtering the audio signal.

Finally, an important issue for research is the question of which of the physiological voice characteristics, and the acoustic parameters representing them, are actually used by listeners to infer the underlying emotions ( $\mathrm{see}^{8,9}$ ).

Here, we report a first exploratory study with three professional opera singers, undertaken to elucidate the link between established measures of glottal operation, as reflected in the waveform and spectrum properties of the FLOGG, and the acoustic parameters as reflected in the long-term-average spectrum (LTAS) of the radiated sound. In particular we examine the following questions: 
1) What are the major FLOGG changes when singers enact a musical sequence with a number of different emotional interpretations?

2) To what extent can these FLOGG changes, difficult to measure in situ or with automatic acoustic analyses, be inferred from LTAS parameters?

3) To what extent do FLOGG and LTAS parameters affect listener judgment of the enacted emotions (and the accuracy of the intended emotional coloring)?

\section{METHOD}

\section{Singers}

A tenor, a baritone, and a bass (T, R and B, respectively) all professional, internationally touring opera singers, accepted to volunteer as subjects. This was particularly advantageous, since such singers have developed an expertise in creating emotional expressivity in singing. They sang in sound treated studio rooms major diatonic scales on the vowel /ae/ or /a/ in a key that comfortably fitted their pitch ranges. They were asked to sing the scale, while imagining that they wanted to project different emotional colors in their interpretation of a lyrical work. The colors they were asked to enact were: Anger, Calm, Contempt, Fear, Joy, Love, Neutral, Pride, Sadness, and Tenderness.

\section{Recording equipment and setting}

The tenor was recorded with an omnidirectional condenser microphone placed on a stand $30 \mathrm{~cm}$ in front of the mouth in a studio at the RTS (Radio Television Suisse) in Geneva. To avoid clipping of loud voice samples and a poor signalto-noise ratio in the soft samples, the audio signal was split into two channels with a $20 \mathrm{~dB}$ amplification difference, and the recording was digitized at a sampling rate of $96000 \mathrm{~Hz}$. The bass and the baritone singers were recorded with a head-mounted omnidirectional microphone in a studio at KTH (Kungliga Tekniska Högskolan, Royal Institute of Technology, Stockholm). On a separate track was recorded an electroglottograph (EGG) signal obtained from a Glottal Enterprises (Syracuse, New York) MC2-1 device. The tracks were digitized and recorded as wav files with a sampling rate of $16000 \mathrm{~Hz}$.

\section{Judgment procedure}

A listening test was run to assess the emotional coloring of the recorded scales. Sixteen voice experts were asked to identify the emotion out of the list of 10 targets (see above) that the respective singer had been asked to enact. Of the listeners, seven were voice teachers, five voice researchers, two vocal coaches, and two professional opera singers. They were all highly experienced in listening to singing in the classical style. Each scale appeared two times in the test, and the stimuli were separated by 6 seconds long pauses. The stimuli were presented in wav files in different random orders to each listener. To estimate the consistency between the judges, we computed the correlations between the profile of correct judgments over all stimuli for each judge with the mean profile of accuracy across all other judges. The mean of these 16 correlations reached $r=0.36$.

\section{FLOGG and LTAS analyses}

The ascending part of the recorded scales were analyzed acoustically in terms of LTAS, using the custom made Sopran software (Svante Granqvist, KTH, software available at www.Tolvan.com) $)^{10}$ ). The frequency range was $0-5000 \mathrm{~Hz}$ and the analysis bandwidth $400 \mathrm{~Hz}$. The following parameters were derived from the LTAS analyses: Alpha ratio (Alpha), Hammarberg Index ( $\left.\mathrm{I}_{\mathrm{Hbg}}\right)$, proportion of energy below $500 \mathrm{~Hz}(\mathrm{E}<.5 \mathrm{kHz})$, proportion of energy below $1000 \mathrm{~Hz}(\mathrm{E}<1 \mathrm{kHz})$, the spectral flatness (Fltns), the spectral centroid (Cntrd), and the spectral slope (Slp). The parameters are defined in Table 1. Further, an attempt was made to estimate the amplitude of the fundamental from the LTAS analysis; for this purpose, another LTAS analysis with a bandwidth of $100 \mathrm{~Hz}$ was run that covered the ascending part of the scale, except for the top pitch. In this way, the first partial of the top pitch was not adding to the amplitude of the second partial of the lowest pitch of the scale. Then, the mean LTAS level in the frequency range covered by the fundamental of the scale and the corresponding mean level one octave higher were measured, and the difference between these means computed. This level difference will be referred to as $\mathrm{H} 1 / \mathrm{H} 2_{\text {LTAS. The }}$ mean sound level of each scale was determined in terms of the equivalent sound level $\left(\mathrm{L}_{\mathrm{Eq}}\right)$ available in the Tools/Histogram module

\section{TABLE 1. \\ LTAS Parameters Analyzed}

\begin{tabular}{|c|c|}
\hline LTAS parameters & \\
\hline Equivalent sound level & $\mathrm{L}_{\mathrm{Eq}}[\mathrm{dB}]$ \\
\hline Alpha ratio & Alpha \\
\hline Level difference between partials 1 and 2 & $\mathrm{H} 1-\mathrm{H} 2_{\text {LTAS }}[\mathrm{dB}]$ \\
\hline Hammarberg index & $\mathrm{I}_{\mathrm{Hbg}}[\mathrm{dB}]$ \\
\hline Proportion energy below $1000 \mathrm{~Hz}$ & $\mathrm{E}<1 \mathrm{kHz}$ \\
\hline Proportion energy below $500 \mathrm{~Hz}$ & $\mathrm{E}<.5 \mathrm{kHz}$ \\
\hline Spectral centroid & Cntrd $[\mathrm{Hz}]$ \\
\hline Spectral flatness & Fltns \\
\hline Spectral slope & SIp [dB/band] \\
\hline FLOGG parameters & \\
\hline Maximum flow declination rate & MFDR $\left[\mathrm{I} / \mathrm{s}^{2}\right]$ \\
\hline Peak-to-peak pulse amplitude & $\mathrm{Ampl}_{\mathrm{AC}}[\mathrm{I} / \mathrm{s}]$ \\
\hline Amplitude quotient & $\mathrm{AO}[\mathrm{ms}]$ \\
\hline Normalized amplitude quotient & NAQ \\
\hline Closed quotient & $\mathrm{Q}_{\text {Closed }}$ \\
\hline Speed quotient & $\mathrm{Q}_{\text {Speed }}$ \\
\hline Level difference between partials 1 and 2 & $\mathrm{H} 1 / \mathrm{H} 2[\mathrm{~dB}]$ \\
\hline $\begin{array}{l}\text { Pitch level, fundamental frequency }\left(f_{\mathrm{o}}\right) \text { measured in } \\
\text { rithmic scale, using } f_{\mathrm{o}}=27.5 \mathrm{~Hz} \text { as reference }\end{array}$ & \\
\hline Alpha Ratio, ratio of the summed energy from $1-5 \mathrm{kh}$ & and \\
\hline $\begin{array}{l}\text { Hammarberg Index, level difference between the } \\
\text { in the } 0-2 \mathrm{kHz} \text { range and the highest spectrum pe }\end{array}$ & peak \\
\hline $\begin{array}{l}\text { Spectral slope } 0-500 \mathrm{~Hz} \text { and } 500-1500 \mathrm{~Hz} \text {, linear } \mathrm{r} \\
\text { spectrum envelope in these given bands. }\end{array}$ & वे ता \\
\hline
\end{tabular}




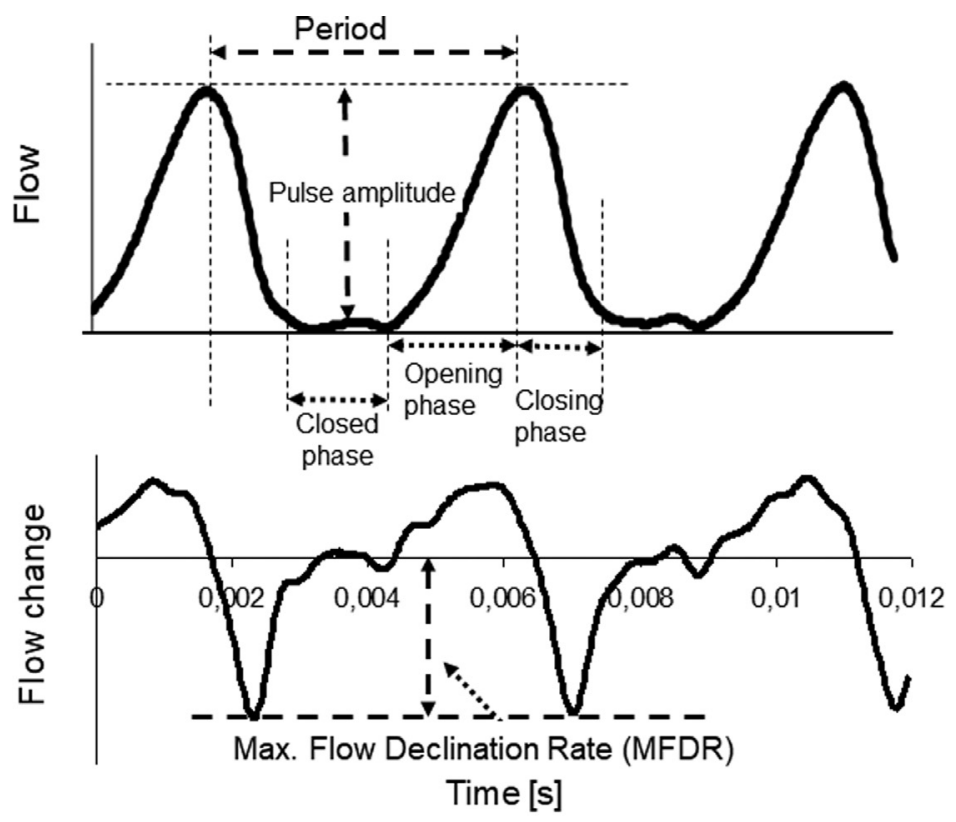

\author{
Quotients \\ $\mathbf{Q}_{\text {closed }}=$ Closed phase $/$ Period \\ $Q_{\text {Speed }}=t_{\text {Opening }} / t_{\text {closing }}$ \\ $A Q$ (Amplitude quotient) $=$ \\ = Pulse amplitude/MFDR \\ NAQ (Normalized amplitude quotient) = \\ $=A Q^{*} f_{0}$
}

FIGURE 1. FLOGG parameters analyzed.

of the Soundswell software (Neovius Data och Signalsystem AB, Lidingö, Sweden).

The FLOGG was analyzed by inverse filtering, using the Sopran software. This software contains both an inverse filter module and a module for analysis of FLOGG parameters. A mid-vowel section of each scale tone was selected for analysis. The filter settings were adjusted manually. For tuning the filters, two criteria were applied: (i) a ripple-free closed phase, and (ii) a source spectrum envelope as free as possible of dips and peaks near the formant frequencies. In setting the filters, the derivative of the EGG signal, after delay of 0.7 milliseconds, was sometimes useful for corroborating that the trailing end of the flow pulse was synchronized with the instant of vocal fold contact. For determining the FLOGG parameters, the Analysis/Glottal flow parameter analysis module of the Sopran software was used. After period, closed phase and peak-to-peak pulse amplitude have been manually marked in the waveform, this software provides and stores in a log file the following FLOGG parameters: fundamental frequency $\left(f_{\mathrm{o}}\right)$, relative duration of the closed phase $\left(\mathrm{Q}_{\text {Closed }}\right)$, peak-to-peak pulse amplitude $\left(\mathrm{Ampl}_{\mathrm{AC}}\right)$, amplitude of the negative peak of the derivative of the FLOGG, ie, the maximum flow declination rate (MFDR), level difference between partials 1 and 2 of the source spectrum $(\mathrm{H} 1 / \mathrm{H} 2)$, amplitude quotient $\mathrm{Ampl}_{\mathrm{AC}}$ MFDR (AQ), AQ normalized with respect to period (NAQ), and duration ratio between the rising and the falling part of the flow pulse $\left(\mathrm{Q}_{\text {speed }}\right)$. These parameters are illustrated in Figure 1.

\section{RESULTS}

In a first step, based on the raw LTAS and FLOGG measures, standardized z-scores were computed separately for each singer (within person standardized z-scores, using the mean and SD across the emotion renderings separately for each singer). This is justified to allow the comparison of emotion enactment effects on the measures, in spite of the differences in basic voice characteristics and vocal characteristics between the three singers. In addition, these personalized z-scores (_Pz) allow direct comparison of measures that are based on highly diverse dimensions and scales.

\section{Differentiation of individual emotions}

To address question 1 in the Introduction, we computed univariate ANOVAs with posthoc comparisons (Duncan criterion); the ANOVA results indicate to which extent different measures can explain the variance between emotions (including significance levels) that the singers intended to convey. The posthoc tests for the existence of significantly different homogeneous subgroups as differentiated by the respective measure. The data are shown in Table 2.

The results show that the FLOGG parameters MFDR, $\mathrm{AQ}, \mathrm{H} 1 / \mathrm{H} 2$, and $\mathrm{Q}_{\mathrm{Closed}}$ make a significant contribution to the discrimination of the intended emotion productions. On the whole, the results for homogeneous subgroups suggest that the most important distinction is between 1) anger, contempt, joy, and pride (and in some cases love) and 2) fear, tender, and sad. Group 1 is characterized by high MFDR and $\mathrm{Q}_{\mathrm{Closed}}$ and low AQ and $\mathrm{H} 1 / \mathrm{H} 2$, whereas the opposite is true for group 2. As to the LTAS parameters, almost all (except $\mathrm{H} 1 / \mathrm{H} 2_{\text {LTAS }}$ ) make significant contributions to variance explanation, with partial eta ${ }^{2}$ (the ratio of variance accounted for) between 0.5 and 0.7. Again, the most important distinction shown by the homogenous subgroups is between 1) anger, contempt, joy, pride (and sometimes love) and 2) fear, tender, and sad on the other. Group 1 shows high $\mathrm{L}_{\mathrm{Eq}}$, Alpha, Fltns, Cntrd, Slp, and low $\mathrm{I}_{\mathrm{Hbg}}, \mathrm{E}<.5 \mathrm{kHz}$, $\mathrm{E}<1 \mathrm{kHz}$, whereas the opposite is true for Group 2. 
TABLE 2.

\section{ANOVA of the FLOGG and LTAS Data}

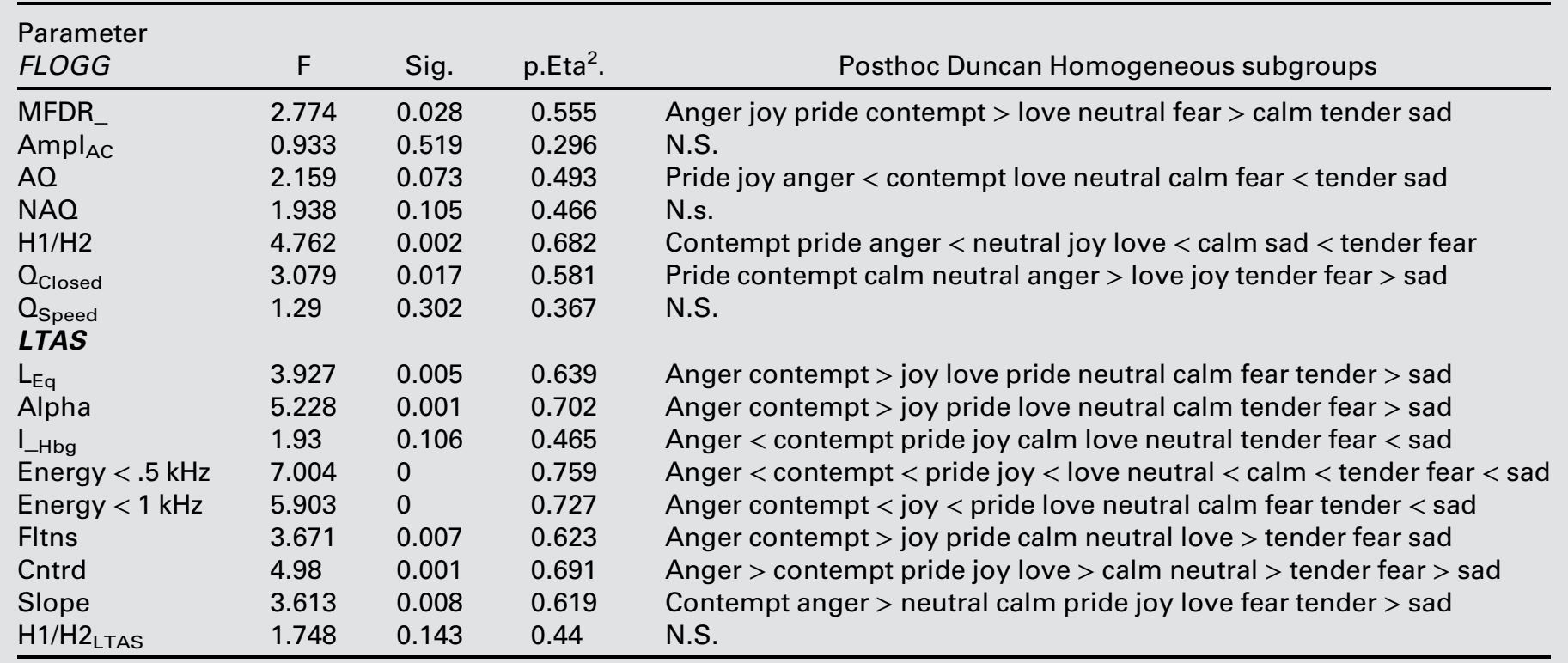

Note: N.S. = no significant subgroup differentiation found; > signs are used to indicate group boundaries resulting from the Duncan criterion, subgroups are ordered from higher to lower group values on the respective variables, whereas < signs are used indicate that subgroups are ordered from lower to higher group values; the number of groups can vary across variables on the basis of the statistical coefficients found in the analysis.

Thus, both FLOGG and LTAS parameters allow to reliably differentiate major emotion "families" This was to be expected; several of the emotions are close to each other with respect to fundamental affective dimensions such as valence, arousal, and power, thus grouping them into a small number of emotion families $\left(\mathrm{see}^{11}\right)$. Arguably, the results shown in Table 2 suggest the following "families":

\begin{tabular}{ll}
\hline Anger, Contempt & $\begin{array}{c}\text { negative valence, feeling of high } \\
\text { power/coping ability }\end{array}$ \\
Joy, Pride, (Love) & $\begin{array}{c}\text { positive valence, feeling of high } \\
\text { power/coping ability }\end{array}$ \\
Tenderness, Calm & $\begin{array}{c}\text { low level of urgency/arousal, feeling } \\
\text { of medium power/coping ability }\end{array}$ \\
Sadness, Fear & $\begin{array}{c}\text { negative valence, feeling of low } \\
\text { power/coping ability }\end{array}$ \\
\hline
\end{tabular}

In response to our first question, then, we can conclude that the FLOGG parameters assessed in this study do indeed vary to a significant extent with the enactment intentions of the singers. As expected on the basis of our earlier work, ${ }^{7}$ this is also true for the LTAS parameters, which yield rather massive effects. The fact that the LTAS parameters are more powerful than the FLOGG parameters is most likely due to the fact that the former parameters cover both source and formant features, whereas the latter capture only glottal functioning.

To determine the nature of the relationships between the FLOGG and the LTAS parameters, we computed a complete correlation matrix between the two sets of parameters for each subject, separately for each singer, shown in
Table S1 of the Supplementary Online Material (SOM). The correlation coefficients between many of the LTAS and FLOGG parameters are extremely high-reaching a mean of .65 for Singer T, .30 for Singer B, and .76 for Singer R. This seems to be due to strong relationships between several FLOGG and LTAS parameters, especially $\mathrm{E}<.5 \mathrm{kHz}$, and MFDR, with equivalent sound level $\mathrm{L}_{\mathrm{Eq}}$, ie, with vocal loudness.

To demonstrate the central importance of $\mathrm{L}_{\mathrm{Eq}}$, Table 3 shows the Pearson correlations between $\mathrm{L}_{\mathrm{Eq}}$, and both the LTAS and FLOGG parameters, separately for all singers. Although the size of the correlations is somewhat different between singers, potentially indicating different vocal strategies, there is a high degree of overlap. As expected, the MFDR is the major predictor, as it represents the strength with which the vocal tract is excited by the voice source. Other important predictors are NAQ and $\mathrm{Ampl}_{\mathrm{AC}}$. This powerful effect of vocal loudness makes it difficult to interpret the relationships between the FLOGG and the LTAS parameters.

In order to understand the underlying relationships more clearly, we computed partial correlations for FLOGG and LTAS parameters with $\mathrm{L}_{\mathrm{Eq}}$ partialed out, thus controlling for the variance accounted for by Leq. The results are shown in Table 4.

This table provides a response to the second question, ie, how much of FLOGG measures are reflected in the standard LTAS parameters? Considering the fact that, unlike the FLOGG parameters, the LTAS parameters are heavily influenced also by the formants, a great number of correlations could not be expected. The strong correlation between MFDR and the two abduction quotients AQ and NAQ may appear surprising, given the fact that the influence of 
TABLE 3.

Correlations Between $L_{E q}$ and the LTAS and FLOGG Parameters, Separately for the three Singers

\begin{tabular}{|c|c|c|c|}
\hline LTAS parameters & $\mathrm{T}$ & B & $\mathrm{R}$ \\
\hline Alpha & 0.857 & 0.744 & 0.955 \\
\hline Cntrd & 0.878 & 0.603 & 0.904 \\
\hline $\mathrm{E}<.5 \mathrm{kHz}$ & -0.982 & -0.745 & -0.990 \\
\hline $\mathrm{E}<1 \mathrm{kHz}$ & -0.840 & -0.746 & -0.905 \\
\hline Fltns & 0.897 & 0.746 & 0.895 \\
\hline $\mathrm{H} 1 / \mathrm{H} 2_{\text {LTAS }}$ & -0.773 & 0.540 & -0.959 \\
\hline $\mathrm{I}_{\mathrm{Hbg}}$ & -0.616 & -0.593 & -0.866 \\
\hline SIp & 0.863 & 0.800 & 0.857 \\
\hline \multicolumn{4}{|l|}{ FLOGG parameters } \\
\hline $\mathrm{Ampl}_{\mathrm{AC}}$ & 0.717 & 0.903 & 0.731 \\
\hline AO & -0.910 & -0.466 & -0.973 \\
\hline $\mathrm{H} 1 / \mathrm{H} 2$ & -0.751 & -0.238 & -0.964 \\
\hline MFDR & 0.954 & 0.783 & 0.945 \\
\hline NAO & -0.900 & -0.331 & -0.972 \\
\hline $\mathrm{Q}_{\text {Closed }}$ & 0.664 & -0.190 & 0.959 \\
\hline $\mathrm{Q}_{\text {Speed }}$ & 0.706 & 0.026 & 0.778 \\
\hline
\end{tabular}

Note: $\mathrm{T}$ - tenor; $\mathrm{B}$ - bass; $\mathrm{R}$ - baritone.

$\mathrm{L}_{\mathrm{Eq}}$ was partialed out and $\mathrm{L}_{\mathrm{Eq}}$ is strongly correlated with MFDR. A reason may be that the relation between MFDR and these parameters is not linear, while the controlling for $\mathrm{L}_{\mathrm{Eq}}$ assumed a linear relation. Also relevant could be that $\mathrm{L}_{\mathrm{Eq}}$ is determined not only by MFDR but affected also by the formant frequencies. This may explain the MFDR influence remaining after controlling for $\mathrm{L}_{\mathrm{Eq}}$. The strong correlation between AQ and NAQ is trivial, since these parameters differ only with respect to the normalization with respect to $f_{\mathrm{o}}$. Both are related to the degree of glottal abduction, a parameter reflected also by the amplitude of the FLOGG fundamental. ${ }^{12}$ Hence, the positive correlation between $\mathrm{H} 1 /$ $\mathrm{H} 2$ and these abduction parameters was expected. ${ }^{13,14}$ Likewise, the correlation between $\mathrm{E}<.5 \mathrm{kHz}$ and $\mathrm{H} 1 / \mathrm{H} 2$ could be expected, as a strong voice source fundamental should affect the LTAS level in the low frequency range, where the fundamental was residing in the present experiment. A lengthening of the closed phase tends to make the FLOGG waveform less similar to a sinewave, which is in accordance with the negative correlations between $\mathrm{Q}_{\mathrm{Closed}}$ and $\mathrm{AQ}$, and NAQ as swell as with $\mathrm{H} 1 / \mathrm{H} 2$. H1/H2 $2_{\text {LTAS }}$ is correlated not only with its FLOGG cousin $\mathrm{H} 1 / \mathrm{H} 2$, but also with all other FLOGG parameters except MFDR and $\mathrm{Ampl}_{\mathrm{AC}}$. These are controlled mainly by subglottal pressure and hence strongly dependent of $\mathrm{L}_{\mathrm{Eq}}$. Summarizing, the observed correlations between the LTAS parameters, particularly $\mathrm{H} 1 / \mathrm{H} 2_{\text {LTAS }}$, and FLOGG parameters, suggest that some information on the phonation type can indeed be extracted from the LTAS analysis.

\section{Reducing measures to major components}

Given the high intercorrelations of the parameters in each of the two sets, it seemed advisable to reduce the

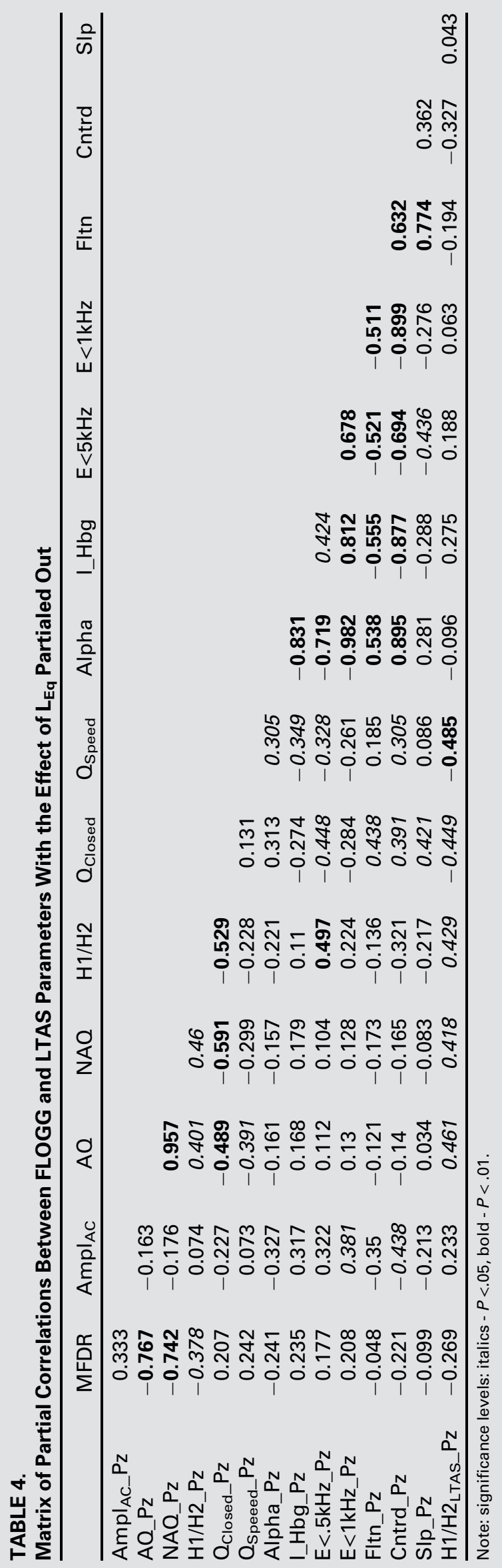


TABLE 5.

\begin{tabular}{|c|c|c|c|c|}
\hline & \multicolumn{4}{|c|}{ Component } \\
\hline & 1 & 2 & 3 & 4 \\
\hline AmplAC & 0.918 & & & \\
\hline MFDR & 0.811 & & & \\
\hline $\mathrm{AO}$ & -0.674 & 0.620 & & \\
\hline NAQ & -0.637 & 0.688 & & \\
\hline $\mathrm{Q}_{\text {Closed }}$ & & -0.891 & & \\
\hline $\mathrm{H} 1 / \mathrm{H} 2$ & & 0.442 & & 0.780 \\
\hline $\mathrm{Q}_{\text {Speed }}$ & & & 0.936 & \\
\hline
\end{tabular}

number of variables using exploratory principal component analysis (PCA). Table 5 shows the results for the FLOGG parameters.

Component 1 seems related to loudness. As mentioned, MFDR is the main determinant of vocal loudness and is controlled by subglottal pressure. It equals the negative peak amplitude of the FLOGG derivative, so it reflects the speed of flow decrease during glottal closing. For this reason, it must be related to the pulse amplitude. The associations with AQ and NAQ are also expected, since these parameters are built on the ratio between the pulse amplitude and MFDR. Component 2 includes these two ratios, which are related to glottal abduction, plus $\mathrm{Q}_{\text {Closed }}$ and H1/ H2 $2_{\text {LTAS, }}$ also known to vary with glottal abduction. A decrease of glottal adduction (ie, increase of abduction) tends to shorten the closed phase. This makes the FLOGG waveform more sinusoidal, which will increase the relative amplitude of the FLOGG fundamental $\mathrm{H} 1$, so this is in accordance with the positive score of $\mathrm{H} 1 / \mathrm{H} 2$. Component 3 is solely associated with the ratio of the durations of the increasing and decreasing portions of the flow pulse and component 4 with the amplitude of the fundamental. For further analyses we used the component scores for these factors.

The corresponding results for the LTAS scales are shown in Table 6. Again, component 1 seems related to vocal loudness. A loudness increase is associated with a decrease of the overall spectrum slope, ie, with a decrease of the level difference between the low and the high frequency part of the
TABLE 6.

\begin{tabular}{lcccc}
$\begin{array}{l}\text { Rotated Component } \\
\text { Parameters }\end{array}$ & Matrix of Loadings for LTAS \\
\hline & \multicolumn{4}{c}{ Component } \\
\cline { 2 - 5 } & 1 & 2 & 3 & 4 \\
\hline I_Hbg & -0.881 & & -0.481 & \\
E $<1 \mathrm{kHz}$ & -0.783 & & 0.501 & \\
Alpha & 0.760 & & & \\
Cntrd & 0.784 & 0.402 & & \\
Fltns & 0.525 & 0.737 & & \\
E<.5kHz & -0.521 & -0.495 & -0.642 & \\
Slope & & 0.829 & & \\
LEq & & 0.507 & 0.719 & \\
H1/H2 2 LTAS & & & & 0.967 \\
\hline
\end{tabular}

spectrum. ${ }^{15}$ Therefore, increase of loudness should result in a decrease of $\mathrm{I}_{\mathrm{Hbg}}$. The same phenomenon is reflected also in the Alpha measure, in the Cntrd and in the Fltns parameters. Also components 2 and 3 appear related to vocal loudness. This is suggested by the positive scores for $\mathrm{L}_{\mathrm{Eq}}$, for Cntrd and for Fltns. On the other hand, the positive score for Slp in Component 2 contradicts this conclusion. Component 4 appears related to glottal adduction, as this parameter affects the amplitude of the fundamental. Given the strong influence of $\mathrm{L}_{\mathrm{Eq}}$ on all measures, it is not surprising that we find loudness represented in three of the four factors, resulting in cross-loadings across factors. However, each of the factors does have a unique signature in the combination of variables that load very highly on the respective factor only.

We used the component scores generated for each component by the PCA for each observation, to examine the relationship of the two sets/domains in a more parsimonious fashion. The correlations between the LTAS and FLOGG component scores are shown in Table 7 . The strong correlation between the LTAS group $\mathrm{L}_{\mathrm{Eq}},-\mathrm{E}<.5 \mathrm{kHz}$, and the FLOGG group $\mathrm{Ampl}_{\mathrm{AC}}$, -MFDR, -AQ, -NAQ is not surprising, since both groups are strongly influenced by vocal loudness. Similarly, the correlation between $\mathrm{H} 1 / \mathrm{H} 2_{\text {LTAS }}$ and the FLOGG group $\mathrm{AQ},-\mathrm{Q}_{\text {Closed }}$ was expected, as a strong FLOGG fundamental should be produced by glottal abduction and short closed phase. The correlation between

TABLE 7.

Pearson Correlations Between the LTAS and FLOGG Component Scores

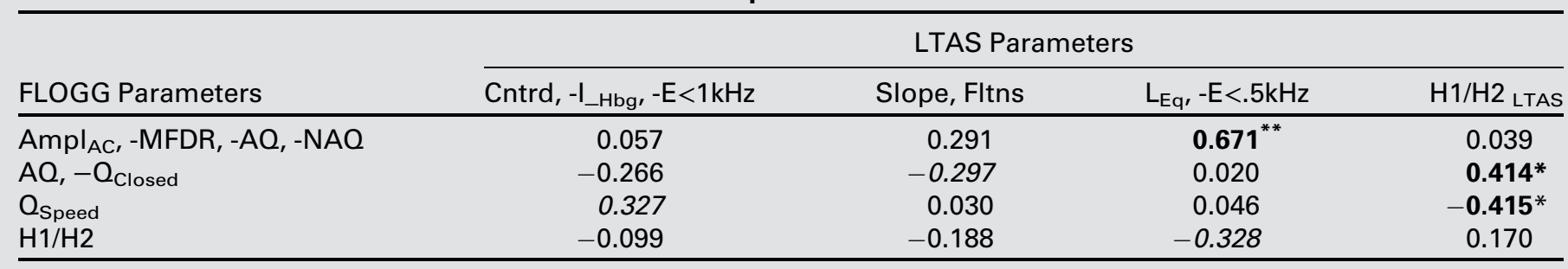

Note: Italics $P<0.10$ ( 0.05 if one-sided = expected), bold $P<0.05$ two-sided. 
TABLE 8

Univariate ANOVAs With Posthoc Comparisons (Duncan Criterion) of the Relations Between the Emotion Families Based on Table 2 and the Groups of FLOGG and LTAS Parameters

\begin{tabular}{lcccl}
\hline FLOGG Parameters & $F$ & Sig. & p.Eta.Sq. & \multicolumn{1}{c}{ Posthoc Duncan Homogeneous Subgroups } \\
\hline AmplAC MFDR -AQ -NAQ & 1.33 & 0.286 & 0.133 & N.s. \\
$(-) Q_{\text {Closed AO }}$ & 3.411 & 0.032 & 0.282 & Fear, Sad > Anger Contempt Tender Calm Neutral Joy Pride Love \\
Q $_{\text {Speed }}$ & 0.747 & 0.534 & 0.079 & N.S. \\
H1/H2 & 3.169 & 0.041 & 0.268 & Fear, Sad $>$ Tender Calm Neutral Joy Pride Love Anger Contempt \\
LTAS parameters & & & & \\
(-)I_Hbg Cntrd -E $<1 \mathrm{kHz}$ & 3.923 & 0.02 & 0.312 & Anger Contempt $>$ Joy Pride Love Tender Calm Neutral Fear, Sad \\
Slope Fltns & 2.218 & 0.11 & 0.204 & N.S. \\
L Eq $-E<.5 \mathrm{kHz}_{\text {H1/H2 LTAS }}^{3.496}$ & 0.03 & 0.287 & Anger Contempt $>$ Joy Pride Love $>$ Tender Calm Neutral Fear, Sad \\
\hline
\end{tabular}

$\mathrm{H} 1 / \mathrm{H} 2_{\text {LTAS }}$ and $\mathrm{Q}_{\text {speed }}$ might suggest that a strong FLOGG fundamental is promoted by a slow opening of the glottis in the vibratory cycle.

\section{Differentiating emotion families}

In the next step, we examined how these component scores fare in discriminating the emotion family groups described above (based on Table 2). As in the case of individual emotions, we computed the univariate ANOVAs with post-hoc comparisons (Duncan criterion) to identify the degree to which the different component score factors allow to discriminate the four emotion families that the singers intended to convey. The results are shown in Table 8.

The results for the FLOGG parameters show that the (-) Qclosed $\mathrm{AQ}$ and the $\mathrm{H} 1 / \mathrm{H} 2$ significantly differentiate the Fear/Sad family from the other three families. In the case of the LTAS parameters, all factors except H1/H2 LTAS significantly differentiate the Anger/Contempt families from the others, as well as showing, in two cases, a significant difference for Fear/Sad at the low end. Both the combined FLOGG and LTAS parameters are very consistent in that the fundamental affective dimension involved in both cases is the high vs low control/power/coping potential dimension. This shows that the FLOGG and LTAS parameters used here have sufficient level of detail to discriminate four emotion families, but that additional parameters might be needed to discriminate individual emotions on an even finer level.

In order to estimate the extent to which these component factors allow to discriminate the four emotion families, we computed a multivariate discriminant analyses for each type of parameter, entering the respective four parameters jointly as predictors. The detailed results are shown in the Supplementary Material. In the case of the FLOGG component factors, $63 \%$ of the stimuli were correctly classified, the most important first discriminant function being characterized by the $\mathrm{Q}_{\text {Closed }}$, AQ and the $\mathrm{H} 1 / \mathrm{H} 2$ factors (see Table S2). For the LTAS parameters, $70 \%$ of the stimuli were correctly classified, the most important first discriminant function being characterized by the Cntrd $\mathrm{E}<1 \mathrm{kHz}$
$\mathrm{I}_{\text {Hbg }}$ factor (see Table S3). These results confirm the pattern shown in Table 8 . The discrimination accuracy in both cases largely exceeds the chance level (one of four factors $=25 \%$ ).

\section{Discrimination of the emotion and emotion families by human listener judges}

The next step is to compare the level of discrimination afforded by the FLOGG and LTAS parameters with the recognition performance of the human listener judges. Given the difficulty of fine discrimination of the individual emotions by means of the vocal LTAS parameters, we would expect that the judges will have had difficulties to precisely recognize individual emotions. This is confirmed by the data in Table 9.

These data confirm earlier expectations. The recognition rates for most of the individual emotions are well above the chance level $(10 \%)$, but still relatively low due to much confusion, particularly within the emotion families described above. This suggests that, just like in the case of the objective parameters, the listeners were better able to differentiate these larger emotion families. This assumption is supported by the confusion matrix for the four emotion families shown in Table 10. The Anger\&Contempt, Joy\&Love\&Pride, and Calm\&Tender\&Neutral families all reached the values in the vicinity of $50 \%$, while the Sad\&Fear family reached only $30 \%$. These percentages are far higher than levels corresponding to random guessing.

\section{DISCUSSION AND CONCLUSION}

In designing the experiment, it was important to create strict experimental conditions. This made the singers' task demanding, quite alien to what they typically encounter in their professional lives; in a real musical setting, a singer can create expressivity by a number of variables other than voice source and average spectrum, eg, tempo, tone onsets and offsets, and micropauses. Indeed, singer T varied tempo considerably, using the slowest tempi for Calm, Tender, Sad and the quickest tempi for Anger, Joy and Fear. In the present experiment, the melodic pattern was fixed, and one singer commented that performing the task felt like biking 
TABLE 9.

Confusion Matrix (in \% Accurate) for the Responses on Individual Emotions to the Listening Test

\begin{tabular}{|c|c|c|c|c|c|c|c|c|c|c|}
\hline Intended & \multicolumn{10}{|c|}{ Rated (\%) } \\
\hline Anger & 47.7 & 20.0 & 10.8 & 0.0 & 12.3 & 0.0 & 0.0 & 1.5 & 0.0 & 7.7 \\
\hline Joy & 10.0 & 8.6 & 31.4 & 0.0 & 34.3 & 2.9 & 0.0 & 7.1 & 0.0 & 5.7 \\
\hline Love & 3.3 & 12.2 & 8.9 & 3.3 & 20.0 & 5.6 & 14.4 & 17.8 & 8.9 & 5.6 \\
\hline Pride & 16.7 & 11.5 & 23.1 & 1.3 & 25.6 & 2.6 & 1.3 & 12.8 & 1.3 & 3.8 \\
\hline Tender & 0.0 & 2.1 & 0.0 & 13.8 & 1.1 & 14.9 & 25.5 & 11.7 & 21.3 & 9.6 \\
\hline Neutral & 4.7 & 16.5 & 5.9 & 3.5 & 5.9 & 10.6 & 5.9 & 35.3 & 2.4 & 9.4 \\
\hline Sad & 1.1 & 8.6 & 0.0 & 12.9 & 3.2 & 14.0 & 28.0 & 6.5 & 14.0 & 11.8 \\
\hline Fear & 4.7 & 2.3 & 7.0 & 5.8 & 7.0 & 3.5 & 20.9 & 15.1 & 7.0 & 26.7 \\
\hline
\end{tabular}

Note: Chance level $=10 \%$, given the random guessing rate for one out of 10 alternatives.

on a two-wheeled bike but being allowed to use only one wheel. On the other hand, the subjects were all professional, classically trained singers, all extremely successful as opera and lieder singers. For this, excellence in creating emotional expressivity also under awkward conditions is crucial in sung performance, particularly in the opera repertoire.

The singers belonged to the classical tradition of opera and lieder singing. Unlike singers in nonclassical styles such as pop, rock, and musical theatre, singers in the classical tradition tend to avoid hyperfunctional voice. This reduced the FLOGG variability in the material analyzed, probably lowering or even eliminating the significance of correlations between the LTAS and FLOGG parameters. Consequently, it would be worthwhile to examine these correlations for more extensive datasets in future research.

The singers used different FLOGG and LTAS characteristics for a given emotion. This is not surprising, as the same emotion can be different in character. For instance, anger can be either hot or cold. The variability also seems to match the fact that very different renderings of the same composition can be rated as perfect. On the other hand, the listening experiment showed that certain combinations of voice characteristics were associated with certain families of emotions.

To allow comparison between LTAS and FLOGG parameters the latter were averaged across the pitches of the scale. Thus, changes of FLOGG parameters along the scale were disregarded and such changes may very well contribute to the emotional color.

Several strong correlations were found among the LTAS parameters, also after controlling for the $\mathrm{L}_{\mathrm{Eq}}$. This means that in the material analyzed the LTAS variation were to a large extent due to variation of vocal loudness. This is partly due to the fact of asking singers to produce vocalizes with a predetermined melody, a scale. In studies on affective speech, there is generally a much larger gamut of parameters that can be varied according to the emotion expressed, producing a larger number of correlations less dependent on $\mathrm{L}_{\mathrm{Eq}}$.

The correlations between LTAS and FLOGG parameters were generally quite high but decreased after controlling for $\mathrm{L}_{\mathrm{Eq}}$. Thus, much of the size of the correlations is in a large part determined by the fact that almost all LTAS and FLOGG parameters are heavily influenced by vocal loudness. Still, a high correlation could have been expected for the $\mathrm{H} 1 / \mathrm{H} 2_{\text {LTAS }}$ and the $\mathrm{H} 1 / \mathrm{H} 2$ derived from the FLOGG; both should reflect the amplitude of the FLOGG fundamental. The reason for the modest correlation would be that this parameter varied with $f_{\mathrm{o}}$ in a way that differed between the emotions. Also, high values are more influential on the averages computed of FLOGG parameters than in the averaging underlying the $\mathrm{H} 1 / \mathrm{H} 2_{\text {LTAS }}$, given that in the latter, the averaging is performed on logarithmic sound level values. In any event, as the low correlations indicate, detailed

TABLE 10.

Confusion Matrix in Percent for the Four Emotion Families Emerging From the Listening Experiment

\begin{tabular}{lccrr}
\hline & Anger\&Contempt & Joy\&Love\&Pride & \multicolumn{1}{c}{ Calm\&Tender\&Neutral } & Sad\&Fear \\
\hline Anger\&Contempt & $\mathbf{5 7 . 1}$ & 33.6 & 3.6 & 5.7 \\
Joy\&Love\&Pride & 20.6 & $\mathbf{4 7 . 9}$ & 22.7 & $\mathbf{8 . 8}$ \\
Calm\&Tende\&rNeutral & 11.4 & 16.1 & $\mathbf{5 1 . 6}$ & $\mathbf{2 0 . 9}$ \\
Sad\&Fear & 8.4 & 17.9 & 44.1 & $\mathbf{2 9 . 6}$ \\
\hline
\end{tabular}

Note: Chance level $=25 \%$, given the random assignment of the rater judgments to one out of four alternatives. 
general inferences regarding the FLOGG are difficult to draw from LTAS parameters alone. For individual speakers, the possibility may be greater, as suggested by the correlations for the individual singers shown in Table S1 in SOM.

With respect to the differences between both types of parameter sets in their covariation with the emotions being expressed by the singers, it is worth noting that here too, the aspect of vocal loudness as linked to MFDR and $\mathrm{L}_{\mathrm{Eq}}$ dominates the patterns of distinctions. Interestingly, the results reported here confirm Scherer's predictions ${ }^{16}$ on Full vs Thin voice; the modal register associated with low $f_{\mathrm{o}}$, high amplitude, strong energy in the entire frequency range as opposed to the head register phonation leading to raised $f_{\mathrm{o}}$ with a relatively low energy of the widely spaced harmonics. These voice patterns were expected as the effect of power and dominance appraisals in the respective emotion episodes. The results reported here in Tables 2 and 8 strongly confirm the 1986 predictions for individual emotions: ${ }^{16}$ from full to thin voice - Anger Contempt $>$ Joy Pride Love Tender Calm Neutral > Fear, Sad.

In the Introduction we formulated three questions. The first concerned the major FLOGG parameters that the singers used to enact a musical sequence with different emotional interpretations. As shown in Table 2, MFDR, ie, vocal loudness, played a major role, but also the parameters $\mathrm{AQ}, \mathrm{H} 1 / \mathrm{H} 2$, and $\mathrm{Q}_{\mathrm{Closed}}$, which all are related to the hyper/ hypo dimension of phonation. Thus, the singers recruited variation of phonation type for enacting different emotions.

The second question was to what extent these FLOGG changes can be inferred from LTAS parameters? It is well known that vocal loudness, or MFDR, has great effects on LTAS. ${ }^{15}$ After partialing out these effects, significant correlations remained between the FLOGG parameters AQ, H1/ $\mathrm{H} 2$, and $\mathrm{Q}_{\text {Closed }}$ and $\mathrm{H} 1 / \mathrm{H} 2_{\mathrm{LTAS}}, \mathrm{E}<.5 \mathrm{kHz}$, and $\mathrm{Slp}$, see Table 4. Thus, it should be possible to derive information about phonation type form LTAS analyses.

The third question was to what extent FLOGG and LTAS parameters affect the listener judgment of the enacted emotions (and the accuracy of the intended emotional coloring). Obviously, $\mathrm{L}_{\mathrm{Eq}}$ is an important parameter. The results also indicated that emotions can be grouped into families and the FLOGG and LTAS parameters into groups. Table 8 suggests that FLOGG and LTAS parameters related to phonation type, $\mathrm{AQ}$ and $\mathrm{Q}_{\text {closed }}$, and the $\mathrm{I}_{\mathrm{Hbg}} \mathrm{Cntrd}$ $\mathrm{E}<1 \mathrm{kHz}$ and the Slp Fltns groups as well as the $\mathrm{H} 1 / \mathrm{H} 2_{\mathrm{LTAS}}$ parameter were all important for the listeners' identification of emotion families.

This study was a first attempt to study the role of FLOGG and LTAS in adding emotional colors to singing. The methods used appear adequate and the results gained seem promising, thus suggesting that it would be worthwhile to undertake large-scale studies, with a larger number of singers (which would also allow to investigate the important studying of the singer-specific correlations between parameters exemplified in the supplementary material).

\section{Acknowledgement}

We are indebted to the singers for their kind and patient cooperation.

\section{SUPPLEMENTARY MATERIALS}

Supplementary material associated with this article can be found in the online version at https://doi.org/10.1016/j. jvoice.2019.08.007.

\section{REFERENCES}

1. Owren MJ, Bachorowski J-A. Measuring emotion-related vocal acoustics. In: Coan JA, Allen JJ, eds. Handbook of Emotion Elicitation and Assessment. Oxford, UK: Oxford University Press; 2007:239-266.

2. Juslin PN, Laukka P. Communication of emotions in vocal expression and music performance: different channels, same code? Psychol Bull. 2003;129:770-814

3. Russell JA, Bachorowski JA, Fernandez-Dols JM. Facial and vocal expressions of emotion. Ann Rev Psychol. 2003;54:329-349.

4. Scherer KR, Johnstone T, Klasmeyer G. Vocal expression of emotion. In: Davidson RJ, Scherer KR, Goldsmith H, eds. Handbook of the Affective Sciences. New York: Oxford University Press; 2003:433456

5. Scherer KR, Clark-Polner E, Mortillaro M. In the eye of the beholder? Universality and cultural specificity in the expression and perception of emotion. Int J Psychol. 2011;46:401-435.

6. Coutinho E, Scherer KR, Dibben N. Singing and emotion. In: Welch G, Howard DM, Nix J, eds. The Oxford Handbook of Singing. Oxford, UK: Oxford University Press; 2019:297-314.

7. Scherer KR, Sundberg J, Fantini B, Trznadel S, Eyben F. The expression of emotion in the singing voice: acoustic patterns in vocal performance. J Acoust Soc Am. 2017;142:1805-1815.

8. Bänziger T, Hosoya G, Scherer KR. Path models of vocal emotion communication. PLoS One. 2015;10: e0136675.

9. Scherer KR, Trznadel S, Fantini B, Sundberg J. Recognizing emotions in the singing voice: a path analytic approach to understanding the process of inference. Psychomusicology. 2017;27:244-255.

10. Tolvan Data, www.tolvan.com, Svante Granqvist. Accessed April 29, 2019.

11. Ekman P. Emotion Families. In: Rauch I, Carr G F, eds. Semiotics around the World: Synthesis in Diversity, Proceedings of the Fifth Congress of the International Association for Semiotic Studies, Berkeley, 1994. Berlin-New York: Mouton de Gruyter; 1997:191-193. (Approaches to Semiotics).

12. Millgård M, Sundberg J, Fors T. Flow glottogram characteristics and perceived degree of phonatory pressedness. J Voice30: 287-292.

13. Sundberg J, Gauffin J. Waveform and spectrum of the glottal voice source. In: Lindblom B, Öhman S, eds. Frontiers of Speech Communication Research. London: Academic Press; 1979:301-320.

14. Alku P, Airas M, Björkner E, Sundberg J. An amplitude quotient-based method to analyze changes in the shape of the glottal pulse in the regulation of vocal intensity. J Acoust Soc Am. 2006;120:1052-1062.

15. Nordenberg M, Sundberg J. Effect on LTAS on vocal loudness variation. Logoped Phoniatr Vocol. 2004;29:183-191.

16. Scherer KR. Vocal affect expression: a review and a model for future research. Psychol Bull. 1986;99:143-165. 